\title{
RECTIFYING SEPARATED NETS
}

\author{
D. Burago And B. Kleiner
}

\section{Introduction}

In our earlier paper [BuK] (see also McMullen's paper [M]), we constructed examples of separated nets in the plane $\mathbb{R}^{2}$ which are not biLipschitz equivalent to the integer lattice $\mathbb{Z}^{2}$. These examples gave a negative answer to a question raised by $\mathrm{H}$. Furstenberg and M. Gromov.

Furstenberg asked this question in connection with Kakutani equivalence for $\mathbb{R}^{2}$-actions, $[\mathrm{F}]$. Return times for a section of an $\mathbb{R}^{2}$-action form a separated net, and to represent the returns of an $\mathbb{R}^{2}$-action by a $\mathbb{Z}^{2}$-action, one has to have a biLipschitz identification of the return times for each point with $\mathbb{Z}^{2}$ (depending measurably on the point). As was pointed out to us by A. Katok, one can use a standard construction of $\mathbb{R}^{2}$-actions to represent our example as the set of return times for points from a set of positive measure, thus showing that not every section can be used (it is worth mentioning here that an old result of Katok $[\mathrm{K}]$ asserts that every $\mathbb{R}^{2}$-action admits a section whose return times are biLipschitz equivalent to $\mathbb{Z}^{2}$ ).

Gromov's motivation for the question came from large scale geometry, and the definition of quasi-isometries. Two metric spaces are quasiisometric if they contain biLipschitz equivalent separated nets; hence one would like to know if the choice of separated net matters, and if a given space can contain nets which are not biLipschitz equivalent. This question is particularly interesting for spaces with cocompact isometry groups.

The counterexample in $[\mathrm{BuK}]$ was based on a counterexample to another question which had been posed by J. Moser and M. Reimann in the 60's, namely whether every positive continuous function on the plane is locally the Jacobian of a biLipschitz homeomorphism. Using well-known properties of quasi-conformal homeomorphisms, one can actually show that any

D.B. supported by a Sloan Fellowship and NSF grant DMS-9803129. B.K. supported by Sloan Fellowship and NSF grant DMS-9972047. 
function that is not the Jacobian of a biLipschitz homeomorphism cannot be the Jacobian of a quasi-conformal homeomorphism either.

The resolution of the original question suggested several intriguing problems, which we present below. These questions came from our discussions with C. McMullen and several other mathematicians.

1. If two finitely generated groups are quasi-isometric, are they biLipschitz equivalent (see [G, p.23])? Here the groups are assumed to be equipped with word metrics. Several special cases of this question are striking. Can $G \times \mathbb{Z}_{2}$ ever fail to be biLipschitz to $G$ ? More generally: is every finite index subgroup of $G$ biLipschitz to $G$ ? By $[\mathrm{W}]$, [S] (see also [P], $[\mathrm{B}])$ it is known that the answers are always affirmative when the group is nonamenable.

2. If $G_{1}$ and $G_{2}$ are uniform lattices in the same connected Lie group, must they be biLipschitz equivalent? (Logically speaking, this is a subcase of problem 1.) Again this is known in the nonamenable case, i.e. the case of nonsolvable Lie groups. Lattices with the same covolume are biLipschitz equivalent by an argument using Hall's marriage lemma (see Lemma 4.1). A similar argument shows that the problem has an affirmative solution when the Lie group admits biLipschitz homeomorphisms with constant Jacobian $t$ for every $t>0$. For instance, graded nilpotent Lie groups and the isometry group of 3-dimensional Solv geometry admit biLipschitz automorphisms which scale volume by an arbitrary factor.

3. If one forms a separated net in the plane by placing a point in the center of each tile of a Penrose tiling, is the resulting net biLipschitz equivalent to $\mathbb{Z}^{2}$ ? More generally, one can consider nets constructed as follows. Let $P \subset \mathbb{R}^{n}$ be a 2-plane with irrational slope, and let $B \subset \mathbb{R}^{n}$ be a bounded subset with nonempty interior. Take the set of points $z \in \mathbb{Z}^{n}$ for which the intersection $(z+B) \cap P$ is nonempty, and project it orthogonally to $P$. When $B$ has small diameter this example can also be described dynamically as the set of return times for a linear $\mathbb{R}^{2}$-action on an $n$-torus to a section.

4. How can one characterize Jacobians of biLipschitz homeomorphisms $\mathbb{R}^{2} \rightarrow \mathbb{R}^{2}$ ? Several authors have studied the prescribed Jacobian problem in other regularity classes $[\mathrm{R}],[\mathrm{DM}],[\mathrm{RiY}],[\mathrm{Y}]$. This question is already nontrivial if one restricts one's attention to nonconstant functions which are locally constant on the complement of a simple closed curve; for instance it seems plausible that in the case of a closed snowflake curve such a function is never the Jacobian of a biLipschitz homeomorphism. On the other hand it seems likely that a function that assumes one value on the subgraph of 
a continuous function $\mathbb{R} \rightarrow \mathbb{R}$ and another value elsewhere is always the Jacobian of a biLipschitz homeomorphism. One can reduce the $n=3$ case of problem 3 to a situation similar to this.

In the remainder of this paper, we settle the $n=3$ case of problem 3 for planes with Diophantine slope. More precisely, let $\alpha \in \mathbb{R}$ satisfy the Diophantine condition

$$
\left|\alpha-\frac{p}{q}\right|>\frac{C}{q^{d}}
$$

for some $C>0, d>2$ and all $p, q \in \mathbb{Z}$; recall that the set of such $\alpha$ has full measure, and contains all algebraic irrationals. Now take $P \subset \mathbb{R}^{3}$ to be the graph of the linear function $\alpha x+\beta y$. Let $X \subset P$ be the separated net obtained by projecting the set $\left\{z \in \mathbb{Z}^{3}:(z+B) \cap P \neq \emptyset\right\}$ orthogonally to $P$.

Theorem 1.2. $X$ is biLipschitz homeomorphic to $\mathbb{Z}^{2}$.

We prove Theorem 1.2 via a general criterion which shows that a separated net is biLipschitz to $\mathbb{Z}^{2}$ provided its density in large subsets approaches a limiting value rapidly enough:

Theorem 1.3. Suppose $Y \subset \mathbb{R}^{2}$ is a separated net. For $\rho>0$ and each measurable subset $U \subset \mathbb{R}^{2}$, define $e_{\rho}(U)$ to be the density deviation

$$
\max \left(\frac{\rho|U|}{\#(U \cap Y)}, \frac{\#(U \cap Y)}{\rho|U|}\right) .
$$

Then define $E_{\rho}: \mathbb{N} \rightarrow \mathbb{R}$ by letting $E_{\rho}(k)$ be the supremum of the quantities $e_{\rho}(U)$, where $U$ ranges over all squares of the form $[i, i+k] \times[j, j+k]$ for $i, j \in \mathbb{Z}$. If there exists a $\rho>0$ such that the product $\prod_{m} E_{\rho}\left(2^{m}\right)$ converges, then $Y$ is biLipschitz to $\mathbb{Z}^{2}$.

REMARK 1.5. The technique from $[\mathrm{BuK}]$ can be used to produce separated nets which have uniform asymptotic density (i.e. $\lim _{k \rightarrow \infty} E(k)=1$ ), but which are not biLipschitz to $\mathbb{Z}^{2}$.

In outline, the proof of Theorem 1.3 goes as follows. First we associate a Voronoi type tiling with the net, and then introduce a function $u$ whose value on each tile is the reciprocal of the area of the tile. We then use Hall's marriage lemma to show that the net is biLipschitz to $\mathbb{Z}^{2}$ if the function $u$ is the Jacobian of a biLipschitz homeomorphism $\mathbb{R}^{2} \rightarrow \mathbb{R}^{2}$. To construct such a homeomorphism, we let $\mathcal{S}_{i}$ be the image under scaling by $2^{i}$ of the usual tiling of $\mathbb{R}^{2}$ by unit squares, and then we let $u_{i}: \mathbb{R}^{2} \rightarrow \mathbb{R}$ be a function whose value of each square of $\mathcal{S}_{i}$ is equal to the average of $u$ over that square. We define a sequence of biLipschitz homeomorphisms $\phi_{i}: \mathbb{R}^{2} \rightarrow \mathbb{R}^{2}$ such 
that $\operatorname{Jac}\left(\phi_{i}\right)=u / u_{i}$, and we show that $\phi_{i}$ subconverges to a biLipschitz homeomorphism $\phi$ by showing the the infinite product $\prod_{i} \operatorname{biLip}\left(\phi_{i} \circ \phi_{i-1}^{-1}\right)$ converges. The biLipschitz homeomorphism $\phi$ has Jacobian $u / \rho$, and we get the desired homeomorphism by composing $\phi$ with the scaling $x \mapsto \sqrt{\rho} x$.

The paper is organized as follows. In section 2 we show in Corollary 2.2 that nets in Theorem 1.2 satisfy the hypotheses of Theorem 1.3. In section 3 we give a sufficient condition for a function $u: \mathbb{R}^{2} \rightarrow \mathbb{R}$ to be the Jacobian of a biLipschitz homeomorphism $\mathbb{R}^{2} \rightarrow \mathbb{R}^{2}$. In section 4 we use the main result from section 3 to prove Theorem 1.3.

Acknowledgements. We would like to thank the anonymous referee for correcting many inaccuracies in the original version of the paper. We are grateful to C. McMullen and G. Margulis for stimulating discussions.

\section{Density Estimates for the Net $X$}

Our objective in this section is to estimate the deviation of the density of $X$ in squares from the asymptotic density of $X$ in $\mathbb{R}^{2}$.

Note that $X$ is biLipschitz to its vertical projection $\bar{X}$ to the $x y$-plane. It is easy to see that in the 3 dimensional situation it is enough to consider the case when $B$ is a ball; indeed, the set of points that we project to $P$ to obtain $X$ is the intersection of $\mathbb{Z}^{3}$ with a slab $W$ parallel to $P$. We will assume that the radius of $B$ is small enough that $W$ does not contain a vertical interval of length 1 . The general case can be reduced to this one by splitting the slab $W$ into a union of thin slabs, and observing that our density estimates are additive. (Finicky readers may note that the discrepancy between the density estimate for closed slabs and for open slabs is negligible.)

Observe that $Z:=W \cap \mathbb{Z}^{3}$ can be represented as the set of points $(x, y, z) \in \mathbb{Z}^{3}$ satisfying $|z-\alpha x-\beta y|<\delta$ for an appropriate $\delta$. Since $W$ does not contain vertical intervals of length 1 , it follows that $\delta<1 / 2, Z$ projects to the $x y$-plane injectively, and hence $\bar{X}$ is biLipschitz homeomorphic to the vertical projection of $Z$ to the $x y$-plane, by a bijection which displaces points by a distance at most $1 / 2$. We denote the projection of $Z$ to the $x y$-plane by $\bar{Z}$. Since there is a bounded displacement bijection between $\bar{Z}$ and $\bar{X}$, the reader may verify that it suffices to obtain density estimates for $\bar{Z}$; the discrepancy between the densities in large squares is due to boundary effects which do not affect the convergence of the product in the statement of Theorem 1.3. 
Pick constants $r, \delta<1 / 2$. Set

$$
S:=\{i \in \mathbb{Z} \mid d(r+i \alpha, \mathbb{Z})<\delta\} .
$$

Proposition 2.1. There are constants $C_{0}$ and $c>0$ such that for any $j$ and $k$,

$$
\left|\frac{1}{k} \#(S \cap[j, j+k))-2 \delta\right| \leq \frac{C_{0}}{k^{c}} .
$$

Let $\rho=2 \delta$, and for any measurable $U \subset \mathbb{R}^{2}$, let $e_{\rho}(U)$ be as in the statement of Theorem 1.3, with $Y=\bar{Z}$.

Corollary 2.2. For all $j_{1}, j_{2} \in \mathbb{Z}, k \in \mathbb{N}$, if $U$ is a square of the form $\left[j_{1}, j_{1}+k\right] \times\left[j_{2}, j_{2}+k\right]$, then $\left|e_{\rho}(U)-1\right| \leq C_{1} / k^{c}$ for some constant $C_{1}$. It follows that the product $\prod_{i} E_{\rho}\left(2^{i}\right)$ converges, where $E_{\rho}$ is defined as in Theorem 1.3.

The corollary can be deduced from the proposition by breaking the square $U$ into rows.

The remainder of this section is devoted to the proof of Proposition 2.1.

Let $p_{n} / q_{n}$ denote the convergents of the irrational number $\alpha$.

Lemma 2.3. For any $n, k,\left|\#\left(S \cap\left[k, k+q_{n}-1\right]\right)-2 \delta q_{n}\right| \leq 3$.

Proof. This is an easy quantative refinement of a standard argument showing that the sequence $\{i \alpha \bmod 1\}$ is equidistributed in the circle $\mathbf{S}=\mathbb{R} / \mathbb{Z}$. We will assume that $\alpha-\frac{p_{n}}{q_{n}}>0$, the other case is absolutely analogous. Consider a finite sequence $x_{i}=\alpha(i+k-1) \bmod 1 \in \mathbf{S}, i=1,2, \ldots q_{n}$. Then \# $\left(S \cap\left[k, k+q_{n}-1\right]\right)$ is nothing but the number of $x_{i}$ 's that belong to the interval of length $2 \delta$ centered at $-r \bmod 1$ in $\mathbf{S}$.

Consider another sequence $y_{i}=(k-1) \alpha+i \frac{p_{n}}{q_{n}} \bmod 1, i=1,2, \ldots q_{n}$. Note that $0<x_{i}-y_{i}<q_{n}\left(\alpha-\frac{p_{n}}{q_{n}}\right)$ (where the first inequality means that $y_{i}$ precedes $x_{i}$ with respect to the natural orientation of $\mathbf{S}$.) This means that there are no other $x_{j}$ on the segment between $y_{i}$ and $x_{i}$. Indeed, otherwise $|(i-j) \alpha-m|<q_{n} \alpha-p_{n}$ for some integer $m$, and this contradicts to the fact that $p_{n} / q_{n}$ is the best approximation for $\alpha$ by rationals with denominators not exceeding $q_{n}$. Hence the points $x_{i}$ and $y_{i}$ alternate in $\mathbf{S}$. This means that there is exactly one member of the sequence $\left\{x_{i}\right\}$ between any two neighboring (with respect to their positions in $\mathbf{S}$, as opposed to their indices) points $y_{i^{\prime}}$ and $y_{i^{\prime \prime}}$.

It is clear that the $q_{n}$ points $y_{1}, y_{2}, \ldots y_{q_{n}}$ are equispaced in $\mathbf{S}$, and hence the number of these points in any interval of length $2 \delta$ differs from $2 q_{n} \delta$ by no more than 1 . Since $x_{i}$ 's and $y_{i}$ 's alternate, the number of $x_{i}$ 's in any interval differs from the number of $y_{i}$ 's in the same interval by no more than two. This completes the proof of the lemma. 
We now return to the proof of Proposition 2.1. Recall that

$$
\left|\alpha-\frac{p_{k}}{q_{k}}\right|<\frac{1}{q_{k} q_{k+1}}
$$

for all $k \geq 0$. Combining this with (1.1), we get

$$
q_{k+1}<C q_{k}^{d-1}=C q_{k}^{h}
$$

where $h:=d-1>1$.

If $l \in \mathbb{Z}$ and $q_{n} \leq l<q_{n+1}$, then from (2.5) we get $q_{n}>(l / C)^{1 / h}$; if moreover $q_{n} \mid l$, we may divide any interval of the form $[j, j+l)$ into $\frac{l}{q_{n}}<C^{\frac{1}{h}} l^{1-\frac{1}{h}}$ intervals of length $q_{n}$, and apply Lemma 2.3 to each of these, getting

$$
|\#(S \cap[j, j+l))-2 \delta l|<C_{1}^{1 / h} l^{1-\frac{1}{h}}=C_{2} l^{h_{1}}
$$

where $C_{2}=C_{1}^{1 / h}, h_{1}=1-\frac{1}{h} \in(0,1)$. Given any interval $[j, j+k)$, let $q_{n}$ be the largest denominator $\leq k$, and set $a:=\left[k / q_{n}\right]$. Then $a q_{n}>k / 2$, and we may apply (2.6) to get

$$
\left|\#\left(S \cap\left[j, j+a q_{n}\right)\right)-2 \delta\left(a q_{n}\right)\right|<C_{2}\left(a q_{n}\right)^{h_{1}}<C_{2} k^{h_{1}} .
$$

Repeating this estimate inductively to the leftover interval $\left[j+a q_{n}, k\right)$, we get that

$$
|\#(S \cap[j, j+k))-2 \delta k|<C_{2}\left(k^{h_{1}}+\left(\frac{k}{2}\right)^{h_{1}}+\ldots\right)=C_{3} k^{h_{1}}
$$

where

$$
C_{3}:=\frac{C_{2}}{1-\left(1 / 2^{h_{1}}\right)} .
$$

\section{A Sufficient Condition for a Function to be a Jacobian}

The main result of this section is:

Proposition 3.1. Let $u: \mathbb{R}^{2} \rightarrow \mathbb{R}$ be a positive function which is constant on each open unit square with vertices in $\mathbb{Z}^{2}$, and let $\rho>0$ be given. For any square $S$ in $\mathbb{R}^{2}$, let $e(S)$ be the quantity

$$
\max \left\{\frac{\rho}{\frac{1}{|S|} \int_{S} u}, \frac{\frac{1}{|S|} \int_{S} u}{\rho}\right\}
$$

where $|S|$ denotes the area of the square $S$. Define an "error" function $E: \mathbb{N} \rightarrow \mathbb{R}$ by letting $E(k)$ be the supremum of $e(\cdot)$ over the collection of $k \times k$ squares of the form $[i, i+k] \times[j, j+k]$, where $i, j \in \mathbb{Z}$. If the product

$$
\prod_{i} E\left(2^{i}\right)
$$


converges, then there is a biLipschitz homeomorphism $\phi: \mathbb{R}^{2} \rightarrow \mathbb{R}^{2}$ with $J a c(\phi)=u$ a.e.

The proof of Proposition 3.1 is based on the following:

Proposition 3.2. There is a constant $C_{1}$ with the following property. Let $T:=[0,2] \times[0,2] \subset \mathbb{R}^{2}$, and let $u: T \rightarrow(0, \infty)$ be a function which is constant in each square $(i, i+1) \times(j, j+1), i, j=0,1$. Then there is a biLipschitz homeomorphism $\phi: T \rightarrow T$ which fixes $\partial T$ pointwise, so that $J a c(\phi)=\lambda u$ a.e., where $1 / \lambda=1 /|T| \int_{T} u$, and $\operatorname{BiLip}(\phi) \leq$ $(\max u / \min u)^{C_{1}}$.

To prove Proposition 3.2, we will need two lemmas.

Let $\|\cdot\|_{\infty}$ denote the $l^{\infty}$ norm on $\mathbb{R}^{2}$, so $\|(x, y)\|_{\infty}:=\max (|x|,|y|)$. Let $S_{r}$ denote the square $\left\{\mathbf{x} \in \mathbb{R}^{2} \mid\|x\|_{\infty} \leq r\right\}$.

Lemma 3.3. Suppose $u_{1}: S_{r} \rightarrow \mathbb{R}$ and $u_{2}: S_{r} \rightarrow \mathbb{R}$ are a continuous positive functions of $\|\cdot\|_{\infty}$, and $\int_{S_{r}} u_{1}=\int_{S_{r}} u_{2}$. Then there is a biLipschitz homeomorphism $\phi: S_{r} \rightarrow S_{r}$ which fixes $\partial S_{r}$ pointwise, so that $J a c(\phi)=$ $u_{1} / u_{2} \circ \phi$ a.e., and

$$
\operatorname{biLip}(\phi) \leq\left(\frac{\max u_{1}}{\min u_{1}}\right)^{C_{2}}\left(\frac{\max u_{2}}{\min u_{2}}\right)^{C_{2}}
$$

where $C_{2}$ is independent of $u$ and $r$.

Proof. We first treat the case where $u_{2} \equiv 1$ and $\int_{S_{r}} u_{1}=\int_{S_{r}} u_{2}=\left|S_{r}\right|$. Set $u:=u_{1}$. Define $f:[0, r] \rightarrow[0, r]$ by

$$
f(t):=\frac{1}{2}\left(\int_{S_{t}} u\right)^{1 / 2},
$$

so $f(0)=0$ and $f(r)=r$. Now define $\phi: S_{r} \rightarrow S_{r}$ by

$$
\phi(\mathbf{x})=f\left(\|\mathbf{x}\|_{\infty}\right) \frac{\mathbf{x}}{\|\mathbf{x}\|_{\infty}}
$$

when $\|\mathbf{x}\|_{\infty} \neq 0$, and $\phi(0)=0$. The map $\phi$ is differentiable on $S_{r} \backslash\{(x, y) \mid$ $|x|=|y|\}$, and calculation shows that

and

$$
\|D \phi\|(\mathbf{x}) \leq k_{1} \max \left(\frac{f\left(\|\mathbf{x}\|_{\infty}\right)}{\|\mathbf{x}\|_{\infty}}, f^{\prime}\left(\|\mathbf{x}\|_{\infty}\right)\right)
$$

$$
\left\|[D \phi(\mathbf{x})]^{-1}\right\| \leq k_{1} \max \left(\frac{\|\mathbf{x}\|_{\infty}}{f\left(\|\mathbf{x}\|_{\infty}\right)}, \frac{1}{f^{\prime}\left(\|\mathbf{x}\|_{\infty}\right)}\right)
$$

where $k_{1}$ is independent of $u$. These quantities are bounded by $k_{2} \frac{\max u}{\min u}$ where $k_{2}$ is independent of $u$. Hence

$$
\operatorname{biLip}(\phi) \leq k_{2} \frac{\max u}{\min u} .
$$


When $\max u / \min u$ is close to 1 , then one gets that

which implies that

$$
\|(D \phi-I)\| \leq k_{3}\left(\frac{\max u}{\min u}-1\right)
$$

$$
\left\|[D \phi]^{ \pm 1}\right\| \leq\left(\frac{\max u}{\min u}\right)^{k_{4}}
$$

where $k_{3}$ and $k_{4}$ are independent of $u$. Combining this with (3.4) we get that

when $C_{2}$ is sufficiently large.

$$
\operatorname{biLip}(\phi) \leq\left(\frac{\max u}{\min u}\right)^{C_{2}}
$$

In the general case, we set

$$
\bar{u}_{i}:=\frac{u_{i}\left|S_{r}\right|}{\int_{S_{r}} u_{i}}
$$

so $\int_{S_{r}} u_{i}=\left|S_{r}\right|$. Applying the special case above, we obtain biLipschitz homeomorphisms $\psi_{1}: S_{r} \rightarrow S_{r}$ and $\psi_{2}: S_{r} \rightarrow S_{r}$ with $\operatorname{Jac}\left(\psi_{i}\right)=\bar{u}_{i}$ a.e., whose biLipschitz constants satisfy (3.5) (with $u$ replaced by $u_{i}$ ). Then $\phi:=\psi_{2}^{-1} \circ \psi_{1}: S_{r} \rightarrow S_{r}$ has Jacobian $u_{1} / u_{2} \circ \phi$ a.e. and

$$
\operatorname{biLip}(\phi) \leq\left(\frac{\max u_{1}}{\min u_{1}}\right)^{C_{2}}\left(\frac{\max u_{2}}{\min u_{2}}\right)^{C_{2}} .
$$

Lemma 3.6. We use the notation $A\left(r_{1}, r_{2}\right)$ for the annulus $\overline{B\left(0, r_{2}\right)}-B\left(0, r_{1}\right)$. Pick $0<a<b$, and set $A:=A(a, b)$. If $u_{1}: A \rightarrow \mathbb{R}$ and $u_{2}: A \rightarrow \mathbb{R}$ are positive Lipschitz functions with

$$
\int_{A} u_{1}=\int_{A} u_{2}=|A|
$$

then there is a biLipschitz homeomorphism $\phi: A \rightarrow A$ with

$$
J a c(\phi)=\frac{u_{1}}{u_{2} \circ \phi} \quad \text { a.e. }
$$

and

$$
\operatorname{biLip}(\phi) \leq\left[\frac{\max u_{1}}{\min u_{1}}\left(1+\operatorname{Lip}\left(u_{1}\right)\right)\right]^{C_{3}}\left[\frac{\max u_{2}}{\min u_{2}}\left(1+\operatorname{Lip}\left(u_{2}\right)\right)\right]^{C_{3}}
$$

for $C_{3}=C_{3}(b / a)$; moreover, when $\left.u_{1}\right|_{\partial A}=\left.u_{2}\right|_{\partial A}$, then $\phi$ can be chosen to fix $\partial A$ pointwise.

Proof. We first assume that $u_{2} \equiv 1$, and set $u:=u_{1}$. Using polar coordinates, we define $I:[a, b] \times[0,2 \pi] \rightarrow \mathbb{R}$ by

$$
I(r, \theta):=\int_{0}^{\theta} u(r, \bar{\theta}) d \bar{\theta} .
$$


Then define $\phi_{1}: A \rightarrow A$ to be the polar coordinate shear

$$
\phi_{1}(r, \theta):=\left(r, \frac{2 \pi I(r, \theta)}{I(r, 2 \pi)}\right) .
$$

The map $\phi_{1}$ clearly defines a biLipschitz homeomorphism of $A$ and

$$
J a c\left(\phi_{1}\right)(r, \theta)=\frac{2 \pi u(r, \theta)}{I(r, 2 \pi)}
$$

for a.e. $(r, \theta)$. Now define $f:[a, b] \rightarrow[a, b]$ by the relation

$$
\int_{A(a, t)} u(r, \theta)=\operatorname{Area}(A(a, f(t))),
$$

and $\phi_{2}: A \rightarrow A$ by $\phi_{2}(r, \theta)=(f(r), \theta)$. Then $\phi_{2}$ satisfies $\operatorname{Jac}\left(\phi_{2}\right)(r, \theta)=$ $I(r, 2 \pi) / 2 \pi$ for a.e. $(r, \theta)$. So we can set $\phi=\phi_{2} \circ \phi_{1}$, and for a.e. $(r, \theta)$,

$$
\begin{aligned}
\operatorname{Jac}(\phi)(r, \theta) & =\left[\operatorname{Jac}\left(\phi_{2}\right)\left(\phi_{1}(r, \theta)\right]\left[\operatorname{Jac}\left(\phi_{1}\right)(r, \theta)\right]\right. \\
& =\left[\frac{I(r, 2 \pi)}{2 \pi}\right]\left[\frac{2 \pi u(r, \theta)}{I(r, 2 \pi)}\right]=u(r, \theta) .
\end{aligned}
$$

We now estimate $\operatorname{biLip}(\phi)$.

One gets $\operatorname{biLip}\left(\phi_{1}\right) \leq k_{1} \max (\max u / \min u,(1+\operatorname{Lip}(u)))$ where $k_{1}$ is independent of $u$. When $\max u / \min u \approx 1$, then at points of differentiability one has

which gives

$$
\left\|\left(D \phi_{1}-I\right)\right\| \leq k_{2}\left(\frac{\max u}{\min u}-1+\operatorname{Lip}(u)\right)
$$

$$
\left\|\left[D \phi_{1}\right]^{ \pm 1}\right\| \leq\left(\frac{\max u}{\min u}(1+\operatorname{Lip}(u))\right)^{k_{3}},
$$

where $k_{2}$ and $k_{3}$ are independent of $u$. It follows that

$$
\operatorname{biLip}\left(\phi_{1}\right) \leq\left(\frac{\max u}{\min u}(1+\operatorname{Lip}(u))\right)^{k_{4}}
$$

where $k_{4}$ is independent of $u$. One easily gets that

$$
\operatorname{biLip}\left(\phi_{2}\right)<k_{5} \frac{\max u}{\min u},
$$

where $k_{5}$ is independent of $u$, and when $\max u / \min u \approx 1$ then

which implies

$$
\left\|\left(D \phi_{2}-I\right)\right\| \leq k_{6}\left(\frac{\max u}{\min u}-1\right),
$$

$$
\left\|\left[D \phi_{2}\right]^{ \pm 1}\right\| \leq\left(\frac{\max u}{\min u}\right)^{k_{7}}
$$

in this case. Combining (3.8) with (3.9) we get

$$
\operatorname{biLip}\left(\phi_{2}\right) \leq\left(\frac{\max u}{\min u}\right)^{k_{8}}
$$


where $k_{8}$ is independent of $u$. Therefore

$$
\operatorname{biLip}(\phi) \leq \operatorname{biLip}\left(\phi_{1}\right) \operatorname{biLip}\left(\phi_{2}\right) \leq\left(\frac{\max u}{\min u}(1+\operatorname{Lip}(u))\right)^{k_{4} k_{8}} .
$$

We now return to the general case when $u_{2} \not \equiv 1$. Applying the special case above, we get homeomorpisms $\psi_{1}: A \rightarrow A$ and $\psi_{2}: A \rightarrow A$ with $\operatorname{Jac}\left(\psi_{i}\right)=u_{i}$ almost everywhere, whose biLipschitz constants are controlled as in (3.11). Then the composition $\psi:=\psi_{2}^{-1} \circ \psi_{1}$ has Jacobian $u_{1} / u_{2} \circ \psi$ a.e., and satisfies (3.7) with $C_{3}=k_{4} k_{8}$.

It remains only to observe that $\left.\psi_{i}\right|_{\partial A}$ is determined by $\left.u_{i}\right|_{\partial A}$, and therefore $\psi$ will fix $\partial A$ pointwise when $\left.u_{1}\right|_{\partial A}=\left.u_{2}\right|_{\partial A}$.

Proof of Proposition 3.2. We will produce $\phi$ as a composition of homeomorphisms $\psi_{1}, \psi_{2}, \psi_{3}$ which are constructed by applying Lemmas 3.3 and 3.6.

We first introduce some notation. Let $T_{i j}:=(i, i+1) \times(j, j+1)$ for $i, j=0,1$. Let $S_{i j} \subset T_{i j}$ be the square with side length $\sqrt{2}-1$ with the same center as $T_{i j}$; note that $S_{i j}$ is the largest square concentric with $T_{i j}$ which is contained in the ball $B((1,1), 1) \subset T$. Let $S^{\prime}$ be the square of side length $\sqrt{2}$ with center at $(1,1)$; so $S^{\prime}$ is contained in $B((1,1), 1)$ and contains the squares $S_{i j}$. Let $A \subset T$ be the annulus centered at $(1,1)$ with radii $1-\frac{\sqrt{2}}{2}$ and 1; note that $A$ contains the squares $S_{i j}$. Finally, let $S$ be the square of side length $2-\sqrt{2}$ with center $(1,1)$; the "hole" $B\left((1,1), 1-\frac{\sqrt{2}}{2}\right)$ of $A$ is inscribed in $S$.

We may assume that ess inf $u=\min u, e s s \sup u=\max u$, and that $\int_{T} u=|T|$. Set $v_{1}:=u$, and let $m:=\min u$. Let $v_{2}: T \rightarrow \mathbb{R}$ be a continuous positive function satisfying

a. $\int_{T_{i j}} v_{2}=\int_{T_{i j}} v_{1}$ for $i, j=0,1$

b. $v_{2} \equiv m$ on the complement of $\cup S_{i j}$;

c. The restriction of $v_{2}$ to $S_{i j}$ is constant on the boundary of each square concentric with $S_{i j}$; and

d. $\frac{\max v_{2}}{\min v_{2}} \leq\left(\frac{\max u}{\min u}\right)^{k_{1}}$ and $1+\operatorname{Lip}\left(v_{2}\right) \leq\left(\frac{\max u}{\min u}\right)^{k_{1}}$; where $k_{1}$ is independent of $u$. Now let $v_{3}: T \rightarrow \mathbb{R}$ be a continuous positive function satisfying

e. $\int_{T} v_{3}=|T|$

f. $v_{3} \equiv m$ on $S$ and on the complement of $S^{\prime}$;

g. $v_{3}$ is constant on the boundary of each square concentric with $T$;

h. $\frac{\max v_{3}}{\min v_{3}} \leq\left(\frac{\max u}{\min u}\right)^{k_{2}}$ and $1+\operatorname{Lip}\left(v_{3}\right) \leq\left(\frac{\max u}{\min u}\right)^{k_{2}}$; where $k_{2}$ is independent of $u$. Finally, set $v_{4} \equiv 1$. 
We now apply Lemma 3.3 to the restrictions $\left.v_{1}\right|_{T_{i j}}$ and $\left.v_{2}\right|_{T_{i j}}$ and obtain a homeomorphism $\psi_{1}: T \rightarrow T$ with $\operatorname{Jac}\left(\psi_{1}\right)=v_{1} / v_{2} \circ \psi_{1}$ a.e. and

$$
\operatorname{biLip}\left(\psi_{1}\right) \leq\left(\frac{\max u}{\min u}\right)^{C_{2} k_{1}} \text {. }
$$

Since $v_{2}(x)=v_{3}(x)=m \leq 1$ for $x \in T-A$, it follows that $\int_{A} v_{2}=$ $\int_{A} v_{3} \geq|A|$. Hence if we set $\bar{v}_{2}:=v_{2}|A| / \int_{A} v_{2}$ and $\bar{v}_{3}:=v_{3}|A| / \int_{A} v_{3}$, then $\operatorname{Lip}\left(\bar{v}_{2}\right) \leq \operatorname{Lip}\left(v_{2}\right)$ and $\operatorname{Lip}\left(\bar{v}_{3}\right) \leq \operatorname{Lip}\left(v_{3}\right)$. We may therefore apply Lemma 3.6 with $u_{1}=\bar{v}_{2}$ and $u_{2}=\bar{v}_{3}$ to get $\psi_{2}: T \rightarrow T$ with $\operatorname{Jac}\left(\psi_{2}\right)=$ $\bar{v}_{2} / \bar{v}_{3} \circ \psi_{2}=v_{2} / v_{3} \circ \psi_{2}$ a.e. and

$$
\operatorname{biLip}\left(\psi_{2}\right) \leq\left(\frac{\max u}{\min u}\right)^{4 k_{2} C_{3}} .
$$

Finally, we apply Lemma 3.3 with $u_{1}=v_{3}$ and $u_{2}=v_{4}$ to get $\psi_{3}: T \rightarrow T$ with $\operatorname{Jac}\left(\psi_{3}\right)=v_{3} / v_{4} \circ \psi_{3}$ a.e. and

$$
\operatorname{biLip}\left(\psi_{3}\right) \leq\left(\frac{\max u}{\min u}\right)^{k_{2} C_{2}}
$$

Then $\phi:=\psi_{3} \circ \psi_{2} \circ \psi_{1}$ satisfies the requirements of Proposition 3.2 where $C_{1}:=C_{2} k_{1}+4 k_{2} C_{3}+k_{2} C_{2}$.

Proof of Proposition 3.1. We may assume without loss of generality that $\rho=1$, since we may postcompose a biLipschitz homeomorphism $\phi_{0}: \mathbb{R}^{2} \rightarrow \mathbb{R}^{2}$ whose Jacobian satisfies $\operatorname{Jac}\left(\phi_{0}\right)=u / \rho$ with the map $x \mapsto \sqrt{\rho} x$, to get $\phi: \mathbb{R}^{2} \rightarrow \mathbb{R}^{2}$ with $\operatorname{Jac}(\phi)=u$.

For each nonnegative integer $q$, let $u_{q}: \mathbb{R}^{2} \rightarrow \mathbb{R}$ be a function whose value on each open square of the form $\left(m 2^{q},(m+1) 2^{q}\right) \times\left(n 2^{q},(n+1) 2^{q}\right)$, $m, n \in \mathbb{Z}$, is equal to the average of $u$ over that square. For each $q \in \mathbb{N}$, we apply Proposition 3.2 to each of the above squares, to obtain a biLipschitz homeomorphism $\psi_{q}: \mathbb{R}^{2} \rightarrow \mathbb{R}^{2}$ with $\operatorname{Jac}\left(\psi_{q}\right)=v_{q}:=u_{q-1} / u_{q} \circ \psi_{q}$ a.e. and

$$
\operatorname{biLip}\left(\psi_{q}\right) \leq\left(\frac{\max v_{q}}{\min v_{q}}\right)^{C_{1}} \leq\left[E\left(2^{q-1}\right)^{2} E\left(2^{q}\right)^{2}\right]^{C_{1}} .
$$

Then $\phi_{q}:=\psi_{q} \circ \ldots \circ \psi_{1}: \mathbb{R}^{2} \rightarrow \mathbb{R}^{2}$ will have Jacobian

and

$$
\frac{u}{u_{q} \circ \phi_{q}}=\frac{u}{u_{q}}
$$

$$
\operatorname{biLip}\left(\phi_{q}\right) \leq\left[\prod_{i=1}^{q} E\left(2^{i}\right)\right]^{4 C_{1}} \leq\left[\prod_{i=1}^{\infty} E\left(2^{i}\right)\right]^{4 C_{1}}<\infty .
$$

Hence $\operatorname{biLip}\left(\phi_{q}\right)$ is uniformly bounded and we may apply Arzela-Ascoli to get the desired biLipschitz homeomorphism $\phi: \mathbb{R}^{2} \rightarrow \mathbb{R}^{2}$. 


\section{Proof of Theorem 1.3}

For $j \in \mathbb{N}$, let $\mathcal{S}_{j}$ be the tiling of $\mathbb{R}^{2}$ produced by rescaling the standard unit tiling by the factor $1 / 2^{j}$. Pick $i$ large enough that no tile of $\mathcal{S}_{i}$ contains more than one element of $Y$. Now form a tiling $\mathcal{T}=\left\{T_{y}\right\}_{y \in Y}$ whose tiles are indexed by $Y$, where $T_{y}$ is a union of tiles from $\mathcal{S}_{i}$ which are closer to $y$ than to any other point of $Y$. The tiles of $\mathcal{T}$ have uniformly bounded diameters and inradii, and $y$ is an interior point of $T_{y}$ for every $y \in Y$. Let $u: \mathbb{R}^{2} \rightarrow \mathbb{R}$ be a function that takes the value $1 /\left|T_{y}\right|$ on the interior of $T_{y}$, for all $y \in Y$. Observe that if $U \subset \mathbb{R}^{2}$ is a square of side length $k$, then $\int_{U} u$ agrees with $\#(Y \cap U)$ to within an error $\leq c_{1} k$. Hence our assumption on $Y$ implies that $u$ satisfies the hypotheses of Proposition 3.1. Therefore by Proposition 3.1, $u$ is the Jacobian of a biLipschitz homeomorphism. The following lemma then completes the proof of Theorem 1.3.

Lemma 4.1. Let $\mathcal{T}=\left\{T_{i}\right\}_{i \in I}$ be a tiling of the plane by tiles with uniformly bounded diameters and inradii. Let $u: \mathbb{R}^{2} \rightarrow \mathbb{R}$ be the function such that $u(x)=1 /\left|T_{i}\right|$ for a.e. $x \in T_{i}$, and suppose $u=J a c(\phi)$ for some biLipschitz homeomorphism $\phi: \mathbb{R}^{2} \rightarrow \mathbb{R}^{2}$. Let $X=\left\{x_{i}\right\}_{i \in I}$ be a separated net where $x_{i}$ lies in the interior of $T_{i}$ for each $i \in I$. Then $X$ is biLipschitz homeomorphic to $\mathbb{Z}^{2}$.

Proof. This is an application of Hall's marriage lemma, which has been used for similar problems by several authors (see $[\mathrm{M}]$, $[\mathrm{W}]$ ). Consider a bi-partite graph $\Gamma$ whose set of vertices is $\mathbb{Z}^{2} \bigcup X$, and there is an edge between $\left(z_{1}, z_{2}\right) \in \mathbb{Z}^{2}$ and $x_{i} \in X$ iff the intersection of $\phi\left(T_{i}\right)$ with the square $\left[z_{1}-0.5, z_{1}+0.5\right] \times\left[z_{2}-0.5, z_{2}+0.5\right]$ is non-empty. Notice that the area of each set $\phi\left(T_{j}\right)$ is 1 . Hence, for every $k$, any collection of $k$ points in $\mathbb{Z}^{2}$ is connected with at least $k$ elements in $X$, because otherwise $k$ squares of the form $\left[z_{1}-0.5, z_{1}+0.5\left[\times\left[z_{2}-0.5, z_{2}+0.5\right]\right.\right.$ would be entirely covered by less than $k$ tiles of the form $\phi\left(T_{j}\right)$, and this is impossible since the total area of the tiles is at most $k-1$. By the same reason, for every $k$, any collection of $k$ points of $X$ is connected with at least $k$ points in $\mathbb{Z}^{2}$. Hence by Hall's marriage lemma, our graph $\Gamma$ contains a bijection $F: X \rightarrow \mathbb{Z}^{2}$.

Notice that $\phi(X)$ is also a separated net, which is biLipschitz equivalent to $X$. To complete the argument, it is enough to show that $\phi(X)$ in its turn is biLipschitz equivalent to $\mathbb{Z}^{2}$. To see this consider the bijection $F \circ \phi^{-1}: \phi(X) \rightarrow \mathbb{Z}^{2}$. If $F \circ \phi^{-1}\left(\phi\left(x_{i}\right)\right)=\left(z_{1}, z_{2}\right)$, then (by definitions of $F$ and $\Gamma$ ), the distance $\operatorname{dist}\left(\phi\left(x_{i}\right),\left(z_{1}, z_{2}\right)\right)$ is bounded by $\sqrt{0.5}+\operatorname{BiLip}(\phi) \sup \operatorname{diam}\left(T_{i}\right)$. Now it remains to notice that a bijection 
between two separated nets that moves every point by a uniformly bounded distance is a biLipschitz equivalence.

\section{References}

[B] O.V. Bogopolsky, preprint, 1996.

[BuK] D. Burago, B. Kleiner, Separated nets in Euclidean space and Jacobians of bi-Lipschitz maps, Geom. Funct. Anal. 8 (1998), 273-282.

[DM] B. Dacorogna, J. Moser, On a partial differential equation involving the Jacobian determinant, Ann. Inst. H. Poincaré Anal. Non Linéaire 7 (1990), 1-26.

[F] H. Furstenberg, Private communication, 1999.

[G] M. Gromov, Asymptotic invariants of infinite groups, in "Geometric Group Theory, Vol. 2 (Sussex, 1991)", Cambridge Univ. Press, Cambridge (1993), 1-295.

[K] A.B. KАток, The special representation theorem for multi-dimensional group actions, in "Dynamical Systems, Vol. I - Warsaw, Soc. Math. France, Paris 1977", Astérisque 49 (1977), 117-140.

[M] C.T. McMullen, Lipschitz maps and nets in Euclidean space, Geom. Funct. Anal. 8 (1998), 304-314.

[P] P. Papasoglu, Homogeneous trees are bi-Lipschitz equivalent, Geom. Dedicata 54 (1995), 301-306.

[R] H.M. Reimann, Harmonische Funktionen und Jacobi-Determinanten von Diffeomorphismen, Comment. Math. Helv. 47 (1972), 397-408.

[RiY] T. RiviÈRE, D. Ye, Resolutions of the prescribed volume form equation, NoDEA Nonlinear Differential Equations Appl. 3 (1996), 323-369.

[S] O. Schramm, Private communication, 1997.

[W] K. Whyte, Amenability, bi-Lipschitz equivalence, and the von Neumann conjecture, Duke Math. J. 99 (1999), 93-112.

[Y] D. Ye, Prescribing the Jacobian determinant in Sobolev spaces, Ann. Inst. H. Poincaré Anal. Non Linéaire 11 (1994), 275-296.

Dmitri Burago, Mathematics Department, Penn State University, University Park, PA 16802, USA

Bruce Kleiner, Department of Mathematics, University of Michigan, 2074 East Hall, Ann Arbor, MI 48109-1109, USA

Current address: Courant Institute of Mathematical Sciences, 251 Mercer St., New York, NY 10012, USA 